\title{
Occupational Dermatoses and Infections
}

\author{
Wayne F. Peate
}

\section{Occupational Skin Disorders}

Work-related dermatoses are common and costly. They are the most frequent occupational illness (not injury), despite significant underreporting, ${ }^{1}$ and account for $40 \%$ to $65 \%$ of workers' compensation cases. ${ }^{2,3}$ Occupational dermatoses rank first as the cause of lost work days and cause one-fourth of all work days lost. ${ }^{4}$ Occupational skin disease affects workers in any setting and of all ages. Highest risk industries include manufacturing, agriculture, construction, machine tool operation, printing, metal plating, leather work, engine service, and forestry. Work-related skin disease can be divided into four clinical groupings: contact dermatitis, oil acne/folliculitis, pigment abnormalities, and neoplasms.

\section{History}

An occupational history is advised for all patients with skin disease in view of the high prevalence of occupational exposures that cause or exacerbate skin disorders. If this initial inquiry raises an appropriate index of suspicion, the patient should be closely questioned regarding the exact time relation between the skin condition (onset, improvement, and recurrence) and work exposure (the effects of time off and return to work). Occupational diseases are caused by predisposing factors and do not appear at random. A helpful resource is the National Institute for Occupational Safety and Health (1-800356-4674 Mondays-Fridays 9:00-4:30 EST; 1-800-424-9300 for 24-hour emergency service).

The diagnostic occupational history should include the following.

1. Current job: including specific activities involving skin contact with potential hazards and general working conditions (e.g., heat and humidity). A worksite visit is often helpful.
2. Exposures: including a complete list of physical, chemical, and biologic agents. Even work tools (e.g., carbonless copy paper) have been implicated.5,6 Ask the patient to provide any trade or chemical names or Material Safety Data Sheets (employers are required to provide) that might assist in identifying work materials that affect the skin.

3. Effects: including skin diseases in other workers.

4. Control measures: including work hygiene (hand washing, solvents for tool cleanup, showers, and laundry service), protective gloves, sleeves, aprons, creams, shields, and enclosures. The control measure itself may give rise to dermatosis. For example, allergic reactions to gloves (latex and other constituents) have risen following the increasing adoption of universal precautions in the workplace and may occur in as many as $3 \%$ of health workers. ${ }^{7}$

5. Past work: including prior compensation for skin disease.

6. Other exposures: including personal hygiene (bathing frequency; soaps, detergents, or industrial skin cleansers used), household cleaning agents, and hobbies involving epoxy resins, paints, and solvents. Ask about medications, especially the topical sensitizers, such as neomycin, -caine anesthetics, and parabens used as stabilizers in steroid creams.

The history of the illness and the occupational history may reveal a close correlation between the health problem and a specific work exposure known to produce such skin effects. The appearance of the condition may signal the cause; for example, vesicular lesions in a glove-like distribution on the hands or dorsilateral on the fingers suggests contact dermatitis. The remainder of the medical history may be noncontributory, but it is not unusual to discover an underlying skin disease that is exacerbated by occupational exposures such as atopy-eczema and wet work. 


\section{Prevention and Control}

Most occupational skin disease is preventable. As a personal or company physician, the family physician is often asked to make determinations and recommendations in the following areas.

1. What predisposing factors contribute to work-related skin disease on a particular job (e.g., a Celtic fair-skinned construction worker chronically exposed to sunlight)?

2. Should patients with preexisting skin disease be advised to avoid certain work environments (e.g., an atopic hairdresser with an eczematous eruption of the hands whose work keeps the hands wet)?

3. What preventive measures should the worker and employer take on the job (e.g., wearing aprons, sleeves, and gloves impervious to cutting oils to avoid occupational acne).

\section{Avoidance of Exposure}

If an agent in the workplace has been identified as a likely cause of a worker's skin disease, further contact must be avoided until the skin is healed. Workers with irritant and even allergic dermatitis can often remain on the job so long as proper protective measures are taken. However, if ordinary protection cannot be guaranteed or if a reasonable accommodation cannot be made, it may be necessary to recommend placing the worker in another job or work station.

\section{Hygiene}

Many dermatoses can be prevented by improved personal and plant cleanliness. Family physicians can counsel individual workers about personal hygiene, including avoidance of organic solvents (e.g., gasoline) for hand cleansing; use of emollients, hand lotions, and creams after washing; frequent change of clothing; daily showering, rapid removal of oil- and chemical-soaked clothes; use of company laundry facilities or separate washing of worker's clothes at home; not eating, drinking, or smoking in the work area; and use of barrier creams (Stokogard, Kerodex).

\section{Engineering and Administrative Controls}

Employers should be informed that they are responsible for protecting worker's health and that preventing occupational skin disease is cost-effective. Engineering controls include mechanical enclosure, exhaust fans, splash shields, drains, proper ventilation, automation of certain processes to minimize skin contact, change in methods (e.g., use of premixed cement decreases exposure to dichromate dust), and substitution of agents or materials. Administrative controls include rotating workers or providing shortened work periods with irritants to limit exposure, providing separate lockers for work and street clothing, and laundering.

\section{Specific Occupational Dermatoses}

In terms of incidence, irritant and allergic contact dermatitides are the most important and common occupational skin diseases. Some important treatment considerations for work-related conditions are as follows.
1. Strong (absolute) irritants (e.g., strong acids or alkalis, or active metals) can cause chemical burns that may progress to necrosis and erosion. Immediate, continuous, prolonged irrigation with water (sometimes up to 2-3 hours), with removal of contaminated clothing under water, reduces the extent of the injury.

2. Do not use water to remove sodium or potassium metal or for phosphorus, which should be smothered with mineral oil.

3. Never treat alkali burns with acid (vinegar) or acid burns with alkali (baking soda), as it would cause an exothermic reaction.

4. Hydrofluoric acid (HF) burns-common in technology industries such as computer chip manufacture-require special care. HF burns are initially painless but may advance to severe tissue necrosis. Recommended topical preparations and soaks (magnesium sulfate or oxide, or quaternary ammonium salts), though effective if applied immediately, are useless by the time the worker sees the physician. Calcium gluconate $10 \%$ should be injected locally into the burn area; in the case of finger or periungual involvement, it is directly infused into the digital arterial supply. ${ }^{8,9}$

\section{Oil Acne/Folliculitis}

Cutting, lubricating, and food production oils, greases, pitch, coal tar and creosote oils, and roofing and paving petroleum products can cause blockage of the pilosebaceous units with comedones, pustules, and papules, a condition that localized trauma in the workplace can exacerbate. Contact dermatitis from irritants and sensitizers in oils and greases may be associated.

Clinical Features. Contact dermatitis extends beyond the usual acne areas into the regions of heavy oil exposure, occurring most often on the hands, arms, and underparts of the body, e.g., thighs exposed to oil-soaked clothing. The condition may aggravate already existing acne. Secondary infection is common (folliculitis, furuncles, oil boils). Personal hygiene is the most important factor for control.

Treatment and Prevention. The patient is advised to avoid contact with oils and greases. Frequent routine cleansing of skin and daily washing of work clothes are mandatory. Routine acne therapy including oral antibiotics is occasionally indicated. ${ }^{10}$

\section{Chloracne}

Exposure to halogenated hydrocarbons, especially chlorinated aromatic compounds [polychlorinated biphenyls (PCBs) and poly biphenyls (PBBs)] and dioxins can result in epithelial thickening in the pilosebaceous unit, follicular blockage, comedone formation, and eventual replacement of the sebaceous gland by keratinized cysts. Manufacturers of herbicides, cable, and electrical insulation (PCBs) are most susceptible. Family members of workers have also been affected.

Clinical Features. Chloracne appears in the usual acne areas and the malar, temporal, postauricular, umbilical, and scrotal regions. ${ }^{11}$ Occasionally, the whole body is involved. Chloracne occurs 4 to 6 weeks after exposure and is more severe and persistent than oil acne. Systemic involvement, porphyria 
cutanea tarda, hepatic and neurologic disorders (headache, peripheral neuropathy, and cerebellar dyskinesia) can occur. Pruritus is present in $50 \%$ of cases. Multiple closed comedones, pale straw-colored cysts, milia, and papules are found. The process may involve every follicle. More extensive involvement leads to abscesses, hyperpigmentation, and hypertrichosis. ${ }^{11}$

Treatment and Prevention. Patients are advised to avoid inhalation of or skin contact with the offending agents. The condition may persist without further exposure for more than a decade. Topical therapy with retinoic acid, oral antibiotics, dermabrasion, and intralesional steroids are often indicated.

\section{Pigment Changes}

Many alterations in pigmentation are secondary to occupational exposure; most are related to melanin. Others include stains, heavy metals, and postinflammatory changes. Trauma, dermatitis, and burns (chemical or thermal) may cause local hypo- or hyperpigmentation, or sometimes both (dyschromia). In darkskinned workers, pigmentation disorders are more pronounced and resistant.

Hypopigmentation. Work-related causes of hypopigmentation include antioxidants used in rubber objects and gloves, paints, plastics, oils (monobenzyl ether of hydroquinone, catechols, and phenols), and Rhus dermatitis (poison oak and poison ivy).

Treatment of most hypopigmentation is minor; it resolves in time and requires no treatment. Treatment for large areas may include oral psoralens and graded sunlight exposure (PUVA). Smaller areas may be concealed by Dy-O-Derm, Dermablend, or Covermark. The cornerstone of prevention is to advise patients to avoid exposure to offending substances; other measures are described in the general prevention discussion.

Hyperpigmentation (Melanosis, Melanoderma). Increased pigmentation is most often the result of sun exposure of outdoor workers. Other causes include the following.

1. Postinflammatory changes (trauma, burns, dermatitis)

2. Ultraviolet light (tars, pitch, psoralens) and photosensitizers together producing erythema and tanning; seen in those involved in roofing and road construction, pipeline work, and wood preservation

3. Melanosis: localized hyperpigmentation secondary to grinding fluids and cutting oils

4. Chemical causes: vegetables, fruits, coal tar, asphalt, pitch, and petroleum oils

Treatment includes bleaching creams containing hydroquinone compounds, but these creams must be used with caution. Extensive depigmentation may result in a cosmetic disaster. Melanex (Nutragena) has been safely used. Time alone may be adequate if preventive measures are followed.

Prevention includes avoiding exposure to the substance and the use of sunscreens and protective clothing to prevent worsening of the condition and to inhibit photosensitizers.

\section{Occupational Skin Neoplasms}

Skin neoplasms are the second most common occupational dermatosis (ranked second only to contact dermatitis), and their incidence is increasing. ${ }^{12} \mathrm{~A}$ long latency period, often 1 to 25 years after exposure, and co-carcinogenesis (e.g., the interaction of sunlight and tar) are characteristic. A number of workplace agents have been associated: polycyclic hydrocarbons, inorganic metals, arsenicals, ionizing radiation, burns (especially burn scars), trauma, and ultraviolet light. Sunlight (ultraviolet wavelengths $256-320 \mathrm{~nm}$ ) is the most common cause and a frequent promoter for many chemical carcinogens, e.g., coal tar and pitch.

Treatment. Treatment adheres to the usual dermatologic standards. Actinic keratoses respond well to liquid nitrogen cryotherapy and 5-fluorouracil if extensive. Shave biopsy is suggested for any suspected malignant lesion. In the case of suspected carcinoma, scalpel excision, curettage, or liquid nitrogen is used. Specialty referral is advised for recurrent lesions (Mohs' technique is suggested) and melanomas, which require wide excision.

Prevention. In addition to the general prevention techniques already reviewed, physicians should advise effective sun protection (hats, long sleeves, sunscreens) and career counseling for fair-skinned individuals to avoid outdoor work.

\section{Occupational Infections}

Work-related infections affect thousands of workers each year, and many occupational groups are at risk. Although occupational infections involve a host of bacterial, viral, fungal, and protozoal agents, they can be subdivided into two broad categories. ${ }^{13}$

1. Zoonoses, such as anthrax, brucellosis, leptospirosis, Rocky Mountain spotted fever, and tularemia (affecting agricultural, animal, and forestry workers).

2. Nonzoonoses, such as hepatitis B, human immunodeficiency virus (HIV) infection, rubella, and tuberculosis (affecting health care, laboratory, day-care, and waste treatment personnel, as well as biotechnology/recombinant DNA workers)..$^{14}$

Knowledge of common infection sources and the work activities that put the worker at risk allows the physician to incorporate risk-assessment questions and diagnostic tests into the history and physical examination, make an early diagnosis, offer prophylaxis when appropriate, begin appropriate treatment, avoid disease complications, prevent disease spread to coworkers, family, and the community, and initiate protective measures.

\section{Social/Occupational History}

The physician should inquire routinely about the following.

1. Work activities. Careful inquiry may suggest the diagnosis in the patient with persistent fever (brucellosis) or chronic cough ( $\mathrm{Q}$ fever), or it may be life-saving if pulmonary an- 
thrax is present. Ask about the health of coworkers and family members.

2. Direct exposures such as needle sticks and animal bites (ticks: Rocky Mountain spotted fever and Lyme disease; dogs and cats: pasteurellosis and rabies; mosquito: viral encephalitis).

3. Indirect exposures, such as splashes of parturition products, blood, and laboratory specimens.

4. Seasonal onset of symptoms (summer and early fall for Lyme disease and mosquito-borne infections, i.e., viral encephalitides).

5. History. Prior infection with brucellosis confers active and cross immunity to other Brucella species. With tetanus, second infections are possible.

6. Prior studies. Previous serologies (many abattoirs require preplacement brucellosis titers), skin testing (tuberculosis, brucellosis), and chest films (echinococcosis: hydatid cysts) provide a baseline.

7. Immunization status (tetanus, rabies, ${ }^{15}$ tularemia, and anthrax).

\section{Prevention and Control}

Work-related infections can usually be prevented by simple measures and current technology, including personal protection (gloves, goggles, aprons); preventive services (immunization, chemoprophylaxis, screening); education (universal precautions, proper hygiene, wound care); workplace revisions [proper disposal of contaminated needles and infected laundry, proper ventilation (e.g., dusty goat wool and anthrax), and dust control (e.g., coccidioidomycosis)]; and animal control [vaccines, adequate sanitation, proper carcass and offal disposal, isolation of infected animals, deworming dogs (e.g., echinococcosis), and rodent control (e.g., leptospirosis)].

\section{Specific Occupational Infections}

\section{Anthrax}

Although anthrax has a low incidence in the United States and other developed countries, the severity of the pulmonary infection and the occasional epidemic ${ }^{16,17}$ justify the family physician's awareness of this condition. Bacillus anthracis is a gram-positive rod that forms durable spores that persist in bone, hair, hides, fertilizer, and soil for years. Direct skin contact with contaminated animal products, inhalation (especially dusty goat wool), and ingestion are the modes of exposure. Groups at risk include abattoir (slaughterhouse) workers, landscapers, and meat and hide processors.

Signs and Symptoms. An occupational history is critical to timely diagnosis of the rapidly progressing, fatal pulmonary infection (inhalation anthrax or woolsorters' disease), which is caused by inhalation of spores. The prodrome mimics a nonspecific upper respiratory infection with a dry cough, followed by bloody sputum, fever, and malaise. The infection then spreads to the mediastinal lymph nodes, leading to dyspnea, chest pain, high fever, hypoxia, pulmonary edema, and bacteremia (rarely hemorrhagic meningoencephalitis). Death occurs within 2 to 3 days.
Cutaneous anthrax, or malignant pustule, is transmitted through broken skin. Itching develops, and a single (multiple in $10 \%$ of cases) small, brownish red papule appears that crowns into a vesicle with surrounding induration and erythema. The lesion ulcerates and forms a 2- to 5-cm painless black eschar (Greek anthrax: coal), which typically occurs on the exposed areas of the face and arms. Disseminated disease results in lymphadenopathy, septicemia, fever, nausea, vomiting, malaise, hypotension, and meningeal involvement.

Gastrointestinal or ingestion anthrax, caused by inadequately cooked meat contaminated with $B$. anthracis, is unusual in temperate zones. Symptoms include abdominal pain, vomiting, fever with prostration, bloody diarrhea, and occasionally septicemia, mesoadenitis, peritonitis, and intestinal perforation. Anthrax meningitis begins with a primary oropharyngeal mucosal lesion followed by chills, fever, edema, and lymphadenopathy.

Diagnostic Tests. A leukocyte count is often normal with cutaneous anthrax but elevated with pulmonary anthrax. Gram stain of a vesicle smear, unfixed with methylene blue, reveals bacilli with red capsules. With pulmonary anthrax, chest radiographs reveal hemorrhagic mediastinal widening, necrotizing lymphadenitis, and possible pulmonary infiltrate; and $B$. anthracis may be found in a sputum sample. With anthrax meningitis, the organism can be isolated from cerebrospinal fluid (CSF), which is often hemorrhagic. B. anthracis may be cultured or identified by immunofluorescence staining. In the acute setting an anthrax ELISA titer greater than 64 or an ETIB reaction to the protective antigen or lethal factor bands in one or more serum samples suggests the diagnosis.

Treatment. For cutaneous anthrax use parenteral penicillin G ( 2 million to 6 million units daily in divided doses), oral tetracycline (250-500 mg q6h), or erythromycin, for 7 days. Skin defects may require cosmetic surgery. Incision and drainage can cause dissemination and are contraindicated.

Prevention and Control. Control measures include killing spores by processing, dyeing, or disinfection; proper ventilation and dust control; drainage of stagnant ponds; wearing of gloves and respirators; and posting precautionary placards with pictures of typical cutaneous lesions. Consider prophylactic antibiotics for workers exposed to infected animals and the vaccine for high risk workers exposed to imported wool and hides.

\section{Brucellosis}

Human brucellosis is contracted from exposure to the secretions and excretions of animals (most commonly hogs, cattle, goats, sheep, and dogs); contact may occur when milking and handling fetal tissue and placentas, ingesting contaminated food and water, or inhaling aerosolized contaminants. A work history is essential for timely diagnosis. The gram-negative bacilli Brucella abortus, B. canis, B. melitensis, and B. suis enter the host through broken skin or the oropharynx and spread to the lymphatics and bloodstream. Infection by one species of Brucella provides immunity to other members of the genus. Livestock producers and meatpackers are the workers at high risk: One-third to one-half may have a history of a subclinical infection. ${ }^{18}$ 
Signs and Symptoms. The acute stage is similar to influenza: fevers, chills, myalgias, arthralgias, weakness, fatigue, night sweats, headache, and occasional diarrhea. Secondary symptoms include constipation, abdominal pain, anorexia, and weight loss. The febrile phase is unpredictable; intermittent fevers occur for up to a month followed by remission for up to 2 weeks, with recurrences or "undulations" for months to years. Chronic complications include subacute bacterial endocarditis, neuritis, orchitis, osteomyelitis, particularly suppurative spondylitis, cholecystitis, and meningoencephalitis.

Diagnostic Tests. A mild lymphocytosis and elevation of the erythrocyte sedimentation rate are common. Case classification depends on the culture and isolation of Brucella species or a fourfold or greater increase in Brucella agglutination titer between acute and convalescent phase sera obtained more than 2 weeks apart. A positive skin test for Brucella indicates prior infection.

Treatment. Give oral tetracycline $500 \mathrm{mg} / \mathrm{day}$ for 3 weeks; repeat for relapses. Oral rifampin (600-900 mg) and doxycycline (200 mg/day) are given for 6 weeks for bone, cardiac, and meningeal involvement. Alternatives include (1) streptomycin or rifampin combined with trimethoprim-sulfamethoxazole and (2) a tetracycline and chloramphenicol, with or without streptomycin. ${ }^{19}$ For toxemia, consider oral prednisone $20 \mathrm{mg}$ three times a day for 5 to 7 days.

Prevention and Control. See the general Prevention and Control section, above.

\section{Leptospirosis}

Leptospira interrogans enters the human host through abrasions, the conjunctivae, or oral mucosa and spreads through the bloodstream to the kidneys, liver, meninges, and skeletal and myocardial muscle. Sources of infection include contaminated urine from infected domestic and wild animals; infected excreta, soil, and water; and rodent and dog bites. Commonly exposed groups include veterinarians, sugar cane and rice farmers, livestock and abattoir workers, meat inspectors, and trappers. Infections occur most frequently in the southern and western United States during the summer and winter months.

Signs and Symptoms. Subclinical infections are common. Signs and symptoms are variable and include bradycardia, chills, conjunctival suffusion, fevers, headache, jaundice, myalgias, nausea, rash, stiff neck, vomiting, and muscle tenderness. More severe infections cause hemoglobinuria, nephritis, and meningitis. Occasionally, neurologic manifestations, psychiatric disturbances, chronic lethargy, and intrauterine fetal infection occur. The mortality rate is $3 \%$ to $6 \%$ and is highest in the elderly and those who develop icterus. ${ }^{20,21}$

Diagnostic Tests. Renal and liver function tests are abnormal, and thrombocytopenia may occur. Relevant studies include wound, blood, and urine cultures, microscopic agglutination or indirect hemagglutination tests, and immunoglobulin $\mathrm{M}$ enzyme-linked immunosorbent assay (IgM ELISA), which is positive if there has been a recent infection.
Treatment. Oral doxycycline $100 \mathrm{mg}$ twice a day for 7 days is advised; alternatively tetracycline $500 \mathrm{mg}$ q6h for 7 days may be given or penicillin G $1.2 \mathrm{~g}$ IV four times a day.

Prevention and Control. Control measures include avoiding contaminated water; wearing protective gloves and boots; providing animal vaccines, proper sanitation, and treatment for cattle and pigs; and maintaining rodent control. Infected animals should be prevented from reaching water and from urinating in water used by humans.

\section{References}

1. NIOSH: National occupational survey: pilot study for development of an occupational disease surveillance method. University of Washington, Department of Environmental Health: Department of Health, Environment and Welfare; 1975 DHEW Publ. 75-162.

2. Adams RM. Foreward: occupational skin disease. In: Occupational medicine: state of the art reviews, Vol. 1. 1986:xiii.

3. Williamson DM. Occupational skin disorders. Practitioner 1982;226:1285.

4. Bureau of Labor Statistics, U.S. Department of Labor, 1991.

5. Marks JG, Trautlein JJ, Zwilich CW, Demers LM. Contact urticaria and airway obstruction from carbonless copy paper. JAMA 1984;252:1038-40.

6. Buring JE, Hennekens $\mathrm{CH}$. Carbonless copy paper: a review of published epidemiologic studies. J Occup Med 1991;33:486-95.

7. Reported cases of allergic reactions to latex gloves on the rise. Infect Control Hosp Epidemiol 1991;12:504-5.

8. Vance MV, Curry SC, Kunkel DR, Ryan PJ, Ruggeri SB. Digital hydrofluoric acid burns: treatment with intraarterial calcium infusion. Ann Emerg Med 1986;15:890-896.

9. Bracken WM, Cuppage F, McLaury RL, Kirwin C, Klassen CD. Comparative effectiveness of topical treatments for hydrofluoric acid burns. J Occup Med 1985;27:733-9.

10. Adams RM. Prevention, rehabilitation, treatment. In Adams RM, editor. Occupational skin disease. Philadelphia: Saunders, 1990:277.

11. Crow KD. Chloracne. Trans St Johns Hosp Dermatol Soc 1970; 56:79-99.

12. Austin DF, Reynolds P. Occupation and malignant melanoma of the skin. In: Gallagher RP, editor. Epidemiology of malignant melanoma: recent results in cancer research. Berlin: Springer-Verlag, 1986:98-107.

13. Dutkiewicz J, Jablonski L, Olenchock SA. Occupational biohazards: a review. Am J Industr Med 1988;14:605-23.

14. Committee of Hazardous Biological Substances in the Laboratory. Descriptive epidemiology of occupational infections in laboratory workers. In: Biosafety in the laboratory: prudent practices for the handling and disposal of infectious material. Washington, DC: National Academy Press, 1989:8-12.

15. Fekety R. Rabies prevention: the latest guidelines. PA Drug Update 1982; October.

16. Pfisterer RM. An anthrax epidemic in Switzerland: clinical, diagnostic and epidemiological aspects of a mostly forgotten disease. J Suisse Med 1991;121:813-25.

17. Raffle PAB. Anthrax. In: Raffle PAB, Lee WR, McCallum RI, Murray R, editors. Hunter's diseases of occupations. London: Macmillan, 1987:718-24.

18. Fox MD, Kaufman AF. Brucellosis in the United States, 19651975. J Infect Dis 1977;136:312.

19. Drugs of choice for bacterial infections. Med Lett 1990;817:4446.

20. Faine S. Leptospirosis-still here. Med J Aust 1986;144:561.

21. Mumford C, Dudley N, Terry H. Leptospirosis presenting as a flaccid paralysis. Postgrad Med J 1990;66:218-20. 